\title{
Características fisico-químicas e sensoriais da carne de bubalinos Murrah abatidos em diferentes períodos de confinamento ${ }^{1}$
}

\section{Cristiana Andrighetto ${ }^{2}$, André Mendes Jorge ${ }^{3}$, Roberto de Oliveira Roça ${ }^{4}$, Érico Rodrigues ${ }^{2}$, Waldmaryan Bianchini ${ }^{2}$, Caroline de Lima Francisco ${ }^{5}$}

\author{
${ }_{1}$ Pesquisa financiada pela Fapesp e Fundunesp. \\ 2 Pós-graduação em Zootecnia da FMVZ/Unesp-Botucatu. \\ 3 DPEA da FMVZ/Unesp-Botucatu. \\ ${ }^{4}$ DGTA da FCA/Unesp-Botucatu. \\ ${ }^{5}$ Curso de graduação em Zootecnia da FMVZ/Unesp-Botucatu.
}

RESUMO - Objetivou-se avaliar as características físicas, químicas e sensoriais da carne de búfalos abatidos em diferentes períodos de confinamento. Foram utilizados 20 bubalinos da raça Murrah, castrados, descornados, com idade média inicial de 15 meses, abatidos aos 75, 100, 125 ou 150 dias de confinamento. Após o abate, as carcaças foram identificadas e resfriadas por 24 horas. Durante a desossa, foram colhidas amostras do contrafilé entre a $10^{\underline{a}}$ e $11^{\underline{a}}$ costelas para análise das características sensoriais e das perdas por cocção; entre a $11^{\underline{a}}$ e $12^{\underline{a}}$ costelas para avaliação da composição centesimal, do valor calórico, do pH e do índice de fragmentação miofibrilar (IFM); e entre a 12a e 13a costelas para a avaliação do escore de marmorização e da força de cisalhamento. A composição centesimal, o pH, a força de cisalhamento, o valor calórico, as perdas por cocção e o IFM não diferiram estatisticamente entre os períodos de confinamento. O grau de marmorização diferiu entre as idades de abate, uma vez os animais abatidos aos 75 dias de confinamento apresentaram escore de 1,25; os abatidos aos 100 e 125 dias, escore 2; e aqueles abatidos aos 150 dias de confinamento, escore 2,2. Em todos os períodos de confinamento, a carne bubalina foi classificada como macia (média 3,94 kgf) e apresentou com características que não diferiram pelo painel sensorial entre os 75 e 150 dias de confinamento. O abate de bubalinos aos 75 e 150 dias de confinamento não influencia as características sensoriais sabor, aroma e cor da carne bubalina.

Palavras-chave: avaliação sensorial, búfalos de rio, composição centesimal, maciez

\section{Physical-chemical and sensory characteristics of meat from Murrah buffaloes slaughtered at different feedlot periods}

\begin{abstract}
The objective was to evaluate the physical, chemical and sensory of buffaloes meat slaughtered in different periods of feedlot. Twenty castrated, hornless Murrah buffaloes with initial average age of 15 months old were slaughtered at $75,100,125$ or 150 days of feedlot. After slaughter, the carcasses were identified and cooled for 24 hours. During the deboning, samples were taken from striploin between the $10^{\text {th }}$ and $11^{\text {st }}$ ribs for sensory characteristics analyses and cooking losses; between $11^{\text {st }}$ and $12^{\text {nd }}$ ribs for centesimal composition, caloric value, $\mathrm{pH}$ and myofibril fragmentation index (MFI); and between the $12^{\text {nd }}$ and $13^{\text {rd }}$ ribs for marbling score and shear force analysis. Centesimal composition, $\mathrm{pH}$, shear force, caloric value, cooking losses and MFI did not differ among feedlot periods. Marbling score differed among slaughter ages since animals slaughtered at 75 days of feedlot showed marbling score of 1.25 , and that slaughtered at 100 and 125 days, marbling score of 2, and those slaughtered at 150 days of feedlot, score 2.2. In all feedlot periods, buffaloes meat was classified as soft (3.94 kgf), with characteristics that did not differ among sensory panel at 75 and 150 days of feedlot. Buffalo slaughter at 75 and 150 days of feedlot does not influence the sensory characteristics as flavor, aroma and color of Buffalo meat.
\end{abstract}

Key Words: centesimal composition, river water buffalo, sensory evaluation, tenderness

\section{Introdução}

No Brasil, aproximadamente $90 \%$ da carne de búfalo é comercializada como carne bovina (Corrêa \& Tramoso, 2004). Existe o conceito equivocado de que a carne de bubalinos é dura e escura, pois a maioria dos animais destinados ao abate é composta de fêmeas de descarte e de animais velhos.

Para melhorar a comercialização da carne bubalina é preciso incentivar o abate de animais jovens e a caracterização de sua carne, padronizando e criando a identidade do produto que será comercializado. 
O confinamento é uma tecnologia que pode ser empregada na bubalinocultura para aumento dos índices de produtividade, utilizando-se rações balanceadas, que melhoram o ganho de peso e reduzem a idade de abate, com efeitos positivos sobre a qualidade das carcaças e a oferta de carne na entressafra (Jorge, 1999).

Na Região Sudeste, a principal fonte de renda na bubalinocultura é a atividade leiteira (Bernardes, 2006). Nesta atividade, o abate dos bezerros machos constitui alternativa para aumentar a renda do produtor.

A carne de búfalo ainda é pouco estudada, o que torna necessária a realização de pesquisas para conhecimento de suas características físico-quimicas e sensoriais. Este trabalho foi realizado com o objetivo de avaliar as características físicas, químicas e sensoriais da carne de búfalos Murrah oriundos de rebanho leiteiro e abatidos em diferentes períodos de confinamento.

\section{Material e Métodos}

O experimento foi conduzido no confinamento experimental do Departamento de Melhoramento e Nutrição Animal da Faculdade de Medicina Veterinária e Zootecnia, UNESP, Campus de Botucatu, São Paulo.

Foram utilizados 20 bubalinos machos, da raça Murrah com idade média inicial de 15 meses e peso inicial de $207 \pm 8,19$ kgg, contemporâneos e provenientes de rebanho leiteiro, castrados aos 12 meses, descornados na primeira semana após o nascimento e submetidos a controle sanitário contra endo e ectoparasitas.

O período de adaptação à dieta, ao manejo e às instalações experimentais foi de 28 dias. Os animais foram distribuídos aleatoriamente em grupos homogêneos de cinco animais, alocados em quatro baias (céu aberto sem sombra, com $10 \mathrm{~m}$ de largura por $30 \mathrm{~m}$ de comprimento), com bebedouro australiano com capacidade para $1.500 \mathrm{~L}$.

Após o período de adaptação, os bubalinos foram submetidos a quatro manejos, para abate aos 75, 100, 125 ou 150 dias de confinamento. A dieta foi fornecida à vontade, em duas refeições diárias (40\% às 8 h e $60 \%$ às 16 h), como ração completa, em quantidade suficiente para que as sobras dos cochos, em um período de 24 horas, correspondessem de 5 a $10 \%$ da matéria seca fornecida.

Para atendimento das necessidades nutricionais e formulação das dietas, utilizou-se o NRC (1996) para bovinos de corte, com exigências para animais castrados em crescimento. A relação volumoso:concentrado utilizada foi de 30:70 e os alimentos usados nas formulações das rações foram feno de Coast cross, silagem de milho, caroço de algodão, polpa cítrica, silagem de grãos úmidos de milho e núcleo Nutrumin ${ }^{\circledR}$ (Tabela 1 ).

Ao final dos períodos de permanência pré-estabelecidos no confinamento, os animais foram abatidos após jejum de sólidos (16 horas), em frigorífico comercial localizado a $40 \mathrm{~km}$ do confinamento experimental, obedecendo ao fluxo normal do estabelecimento. Ao abate, as carcaças foram identificadas, pesadas e resfriadas por 24 horas a temperatura da câmara de $0^{\circ} \mathrm{C}$.

Após o resfriamento, durante a desossa, foram colhidas amostras de aproximadamente $2,50 \mathrm{~cm}$ do músculo Longissimus dorsi para as análises laboratoriais. As amostras da $10^{\underline{a}}$ à $11^{\underline{a}}$ costela foram destinadas à análise sensorial e à determinação das perdas por cocção e aquelas da $11^{\mathrm{a}}$ à $12^{\mathrm{a}}$ costela foram utilizadas na avaliação da composição centesimal, do valor calórico, do pH e do índice de fragmentação miofibrilar. As amostras foram congeladas $\left(-20^{\circ} \mathrm{C}\right)$ para posterior análise em laboratório do índice de marmorização e da força de cisalhamento.

A análise da força de cisalhamento, para todos os períodos de confinamento, foi conduzida no laboratório do Departamento de Química e Bioquímica do Instituto de Biociências - Unesp, Campus de Botucatu, utilizando-se o procedimento "Warner-Bratzler Shear", proposto por Wheeler et al. (1995).

A determinação do índice de fragmentação miofibrilar (IFM) foi realizada no Laboratório do Departamento de Química e Bioquímica do Instituto de Biociências, UNESPBotucatu, segundo método descrito por Culler et al. (1978).

As análises de composição centesimal foram realizadas no Departamento de Gestão e Tecnologia Agroindustrial da Faculdade de Ciências Agronômicas - Unesp/Botucatu. Nas análises utilizaram-se amostras do músculo Longissimus

Tabela 1 - Composição percentual da dieta, com base na matéria seca (MS), e valores calculados da dieta

\begin{tabular}{lc}
\hline Ingrediente & MS (\%) \\
\hline Silagem de milho & 11,54 \\
Feno de coast cross & 16,14 \\
Caroço de algodão & 3,50 \\
Polpa cítrica peletizada & 10,10 \\
Silagem de grãos úmidos de milho & 46,56 \\
Núcleo NUTRUMIN ${ }^{\circledR}$ & 12,16 \\
Proteína bruta (\%) & 15,0 \\
Energia metabolizável (Mcal/kg) & 2,72 \\
\hline
\end{tabular}

Composição do núcleo NUTRUMIN (em \% da MS): farelo de algodão - 43,56; farelo de soja - 29,18; uréia - 2,9; calcário - 13,62; mineral* - 10,70; rumensin 0,02

* Composição da mistura mineral/kg de produto: P - 75 g; Ca - 126 g; Na 160 g; Cl - 240 g; S - 20 g; Mg - 15 mg; Zn - 4.000 mg; Cu - 1.800 mg; Fe 1.500 mg; Mn - 1.400 mg; Co - 150 mg; I - 120 mg; Se - 15 mg; F - 750 mg. 
dorsi. Foram avaliados: a umidade e os teores de proteína, extrato etéreo e cinzas, segundo métodos da AOAC (1990).

A análise do valor calórico da carne foi realizada no Departamento de Gestão e Tecnologia Agroindustrial da Faculdade de Ciências Agronômicas - Unesp/Botucatu utilizando-se amostras in natura do músculo Longissimus dorsi. O valor calórico foi determinado em bomba calorimétrica, PARR 1281.

$\mathrm{O} \mathrm{pH}$ da carne foi determinado em aparelho da marca Tecnal, no músculo Longissimus dorsi, entre a $11^{\mathrm{a}}$ e a $12^{\underline{a}}$ costela, após as 24 horas de resfriamento da carcaça.

Para a determinação do escore de marmorização, foi utilizada a metodologia descrita pelo USDA Quality Grade (1997), com escore de 1 a 10, em que: 1 = praticamente ausente; 2 = traços; 3 = leve; 4 = pouco; 5 = modesto; 6 = moderado; 7 = levemente abundante; 8 = moderadamente abundante; 9 = abundante; e 10 = muito abundante.

Nos painéis sensoriais, foram avaliadas somente as amostras do primeiro ( 75 dias de confinamento) e do último abate (150 dias de confinamento), uma vez que, no painel sensorial, a utilização de mais que duas amostras de carne dificulta a análise pelo provador. A utilização dos painéis sensoriais (dois) teve como objetivo observar possíveis diferenças nas características sensoriais entre os períodos de confinamento.

Para análise sensorial, as amostras foram mantidas em salmoura $10 \%$ durante 60 minutos a $5^{\circ} \mathrm{C}$, na proporção 1:1. Em seguida, foram acondicionadas em papel-alumínio e submetidas a aquecimento em chapa elétrica pré-aquecida por 30 minutos e regulada para $250^{\circ} \mathrm{C}$. Ao atingirem a temperatura final de $90^{\circ} \mathrm{C}$, medida no centro geométrico, as amostras foram retiradas da chapa.

As avaliações sensoriais foram conduzidas conforme descrito por Meilgaard et al. (1990) e Roça et al. (1988), com dez provadores treinados (Roça \& Bonassi, 1985). As amostras foram aquecidas em forno elétrico de dupla resistência por 5 minutos a $100^{\circ} \mathrm{C}$ e servidas imediatamente aos provadores, em recipiente de aço inoxidável. Foram aplicados os seguintes testes sensoriais: aroma, aroma estranho, sabor, sabor estranho, maciez, suculência, mastigabilidade e cor.

Antes de realizar a salga para a preparação das amostras para análise sensorial, as amostras foram pesadas e foi obtido o peso inicial da amostra, após cocção em chapa elétrica pré-aquecida por 30 minutos, regulada para $250^{\circ} \mathrm{C}$. Ao atingirem a temperatura interna final de $90^{\circ} \mathrm{C}$, as amostras foram retiradas da chapa e pesadas novamente para cálculo das perdas percentuais por cocção.

O delineamento experimental adotado foi o inteiramente ao acaso, com quatro tratamentos e cinco repetições. As médias dos tratamentos para composição centesimal, valor calórico, índice de fragmentação miofibrilar, pH, perdas por cocção e força de cisalhamento foram comparadas pelo teste Tukey.

Para índice de marmorização, aroma estranho, sabor estranho, maciez e suculência, utilizou-se análise de variância não-paramétrica, uma vez que os dados não apresentaram distribuição normal. O teste adotado para marmorização nesse caso foi o Kruskal-Wallis por apresentar mais de duas variáveis, enquanto, para aroma estranho, sabor estranho, maciez e suculência, utilizou-se o teste de Wilcoxon por apresentar duas variáveis. Para os demais parâmetros da análise sensorial, foi utilizada a análise de variância e a diferença entre tratamento foi avaliada pelo teste $\mathrm{F}$.

Calculou-se a correlação entre índice de fragmentação miofibrilar e força de cisalhamento utilizando-se correlações de Pearson. Para todas as análises, foi utilizado o programa SAEG(UFV,1997).

\section{Resultados e Discussão}

Na análise da composição centesimal e do valor calórico, não houve influência $(\mathrm{P}>0,05)$ dos períodos de confinamento sobre a umidade, os teores proteína, gordura e cinzas e o valor calórico (Tabela 2) do músculo Longissimus dorsi.

Os valores observados para umidade, cinzas e proteína corroboram os obtidos por Mattos et al. (1997), que, avaliando animais da raça Mediterrâneo terminados a pasto, obtiveram valores de $74,31 \%$ para umidade, $22,73 \%$ de proteína e 1,08\% de cinzas. Somente para o extrato etéreo, os resultados foram inferiores (0,60\%). Andrighetto et al. (2005) avaliaram o músculo Longissimus dorsi de bubalinos Mediterrâneo não-castrados e abatidos com diferentes pesos e também obtiveram menores valores de extrato etéreo (2,28\%). Os dados da literatura revelaram menor quantidade de extrato etéreo nos animais estudados, por não serem castrados. Segundo Purchas et al. (2002), animais castrados depositam mais gordura intramuscular, o que explica a maior quantidade de gordura observada neste estudo.

O núcleo de estudos e pesquisa em alimentação (NEPAUnicamp, 2004) desenvolveu tabelas de composição de alimentos consumidos no Brasil. Nesse estudo, o valor obtido para contrafilé bovino cru (Longissimus dorsi) sem gordura foi de $157 \mathrm{kcal} / 100 \mathrm{~g}$ de carne. Esse valor é superior aos encontrados em bubalinos em todos os períodos de confinamento, o que comprova que o contrafilé bubalino apresenta menos calorias em comparação ao contrafilé bovino sem gordura. Entretanto, Cédres et al. (2002) obser- 
Tabela 2 - Composição centesimal e valor calórico do músculo Longissimus dorsi de bubalinos da raça Murrah castrados e abatidos em diferentes períodos de confinamento

\begin{tabular}{lrrrr}
\hline Parâmetro & \multicolumn{4}{c}{ Período em confinamento (dias) } \\
\cline { 2 - 5 } & \multicolumn{1}{c}{75} & \multicolumn{1}{c}{100} & \multicolumn{1}{c}{125} & 150 \\
\hline Umidade (\%) & $73,76 \pm 0,56$ & $73,93 \pm 0,56$ & $74,36 \pm 1,65$ & $74,70 \pm 1,08$ \\
Proté́na (\%) & $22,06 \pm 0,33$ & $21,62 \pm 0,43$ & $21,03 \pm 0,73$ & $21,30 \pm 0,76$ \\
Extrato etéreo (\%) & $2,76 \pm 0,42$ & $3,03 \pm 0,58$ & $3,56 \pm 0,90$ & $3,15 \pm 0,42$ \\
Cinzas (\%) & $1,06 \pm 0,03$ & $1,05 \pm 0,03$ & $1,04 \pm 0,05$ & $1,08 \pm 0,05$ \\
Valor calórico (kcal/100 g) & $138,03 \pm 6,00$ & $138,26 \pm 1,92$ & $138,29 \pm 13,22$ & $136,00 \pm 8,25$ \\
\hline
\end{tabular}

* Médias seguidas de letras distintas diferem $(P<0,05)$ entre si.

varam que o valor calórico do contrafilé de bubalinos criados em pasto foi de 101,1 kcal/100g de carne.

A força de cisalhamento, o índice de fragmentação miofibrilar, o pH final e as perdas por cocção do músculo Longissimus dorsi não diferiram $(\mathrm{P}>0,05)$ entre os períodos de confinamento (Tabela 3). A maciez da carne é o fator de maior variabilidade e o atributo mais desejável pelo consumidor. Neste estudo, os valores de força de cisalhamento encontrados nos quatro períodos de confinamento foram inferiores a 5,0 kgf (Tabela 4), o que, segundo Felício (1997), permite classificar a carne como macia.

De acordo com Allingham et al. (1998), a rápida taxa de crescimento reduz a contribuição do tecido conjuntivo e do colágeno responsável pela dureza da carne, motivo pelo qual não se observaram diferenças estatísticas $(P>0,05)$ na maciez da carne com o avanço dos períodos de confinamento.

Andrighetto et al. (2005) constataram valor semelhante para força de cisalhamento (3,55 kgf) em bubalinos Mediterrâneo não-castrados terminados em confinamento e abatidos aos 18 meses de idade.

O índice de fragmentação miofibrilar (IFM) representa a alteração estrutural de miofibrilas naturais, identifica a variação da maciez pela taxa e a extensão da proteólise (Dabés, 2000). Culler et al. (1978) estabeleceram que, para o músculo Longissimus dorsi, índice de fragmentação miofibrilar maior que 60 caracteriza carne bastante macia, enquanto índice abaixo de 60 indica maciez moderada e inferior a 50 indica falta de maciez.
A carne dos animais abatidos em todos os períodos de confinamento (Tabela 3) apresentou índice de fragmentação miofibrilar maior que 60 , confirmando que a carne de bubalinos jovens é macia. Independentemente do período de confinamento, a correlação entre o índice de fragmentação miofibrilar e a força de cisalhamento neste estudo foi de -0,66 $(\mathrm{P}<0,05)$, superior à encontrada em bovinos de corte por Vestergaard et al. (2000), que observaram coeficiente de correlação -0,47, e ao obtido por Manço (2006), que relatou coeficiente de correlação igual a -0,44.

Os valores de $\mathrm{pH}$ encontrados nos períodos de confinamento estudados foram de 5,50 a 5,52. Mattos et al. (1997) verificaram $\mathrm{pH} 5,48$ após 24 horas do abate e Spanghero et al. (2004) encontraram pH de 5,47 no Longissimus dorsi de bubalinos Mediterrâneo.

Segundo Roça (1997), pH final igual a 6,0 é o limite entre o corte normal e o corte escuro característico da carne DFD (dark, firm, dry), mais freqüente em animais não-castrados. Considerando o pH menor que 5,9 como limite para exportação de carne para a União Européia, os valores encontrados para a carne bubalina não seriam limitantes.

Os resultados para perdas por cocção do músculo Longissimus dorsi foram semelhantes aos encontrados por Vaz et al. (2003), também no músculo Longissimus dorsi de bubalinos Mediterrâneo terminados em confinamento. Esses autores observaram 34\% de perdas por cocção na carne de animais alimentados com cana-de-açúcar e 35,3\% na carne dos alimentados com silagem de milho. Spanghero

Tabela 3 - Força de cisalhamento, índice de fragmentação miofibrilar, pH, perda por cocção e escore de marmorização do músculo Longissimus dorsi de bubalinos da raça Murrah castrados e abatidos em diferentes períodos de confinamento

Parâmetro
Período em confinamento (dias)

\begin{tabular}{cccc}
\hline \multicolumn{4}{c}{ Periodo em confinamento (dias) } \\
\hline $4,22 \pm 0,94$ & 100 & 125 & 150 \\
$60,50 \pm 5,83$ & $4,39 \pm 0,61$ & $3,08 \pm 0,95$ & $3,90 \pm 0,59$ \\
& $62,80 \pm 16,45$ & $75,00 \pm 13,24$ & $67,20 \pm 8,07$ \\
$5,52 \pm 0,09$ & $5,52 \pm 0,07$ & $5,52 \pm 0,04$ & $5,50 \pm 0,03$ \\
$35,06 \pm 3,33$ & $39,24 \pm 4,64$ & $33,74 \pm 7,46$ & $37,88 \pm 3,01$ \\
$1,25 \pm 0,45 \mathrm{~b}$ & $2,00 \pm 0,00 \mathrm{a}$ & $2,00 \pm 0,00 \mathrm{a}$ & $2,20 \pm 0,45 \mathrm{a}$ \\
\hline
\end{tabular}

Força de cisalhamento (kgf)

Índice de fragmentação

miofibrilar (\%)

$\mathrm{pH}$ final

Perdas por cocção (\%)

Escore de marmorização

* Médias seguidas de letras distintas diferem $(P<0,05)$ entre si. 
et al. (2004) relataram a ocorrência de 32,7\% de perdas por cocção em estudo com bubalinos da raça Mediterrâneo.

A marmorização ocorre em fase de desenvolvimento mais adiantada, pois a deposição de gordura intramuscular é mais tardia em relação à cobertura de gordura da carcaça (Berg \& Walters, 1983). Neste estudo, os períodos de confinamento influenciaram $(\mathrm{P}<0,05)$ a marmorização da carne, que foi menor na carne dos animais abatidos aos 75 dias de confinamento (Tabela 3). Esses resultados corroboram os encontrados por Tatum et al. (1980), que observaram aumento significativo do escore de marmorização de bovinos aos 160 dias de confinamento em comparação ao obtido aos 100 e 130 dias de confinamento.

Cédres et al. (2002) estudaram o índice de marmorização, também avaliado pela metodologia do USDA (1997), em bubalinos da raça Murrah abatidos com 29 a 32 meses de idade, e observaram escore de marmorização de 1,5. Nesta pesquisa, no entanto, o resultado obtido em amostras de carne dos animais abatidos aos 100,125, 150 dias de confinamento foi superior ao descrito por esses autores, que estudaram animais não-castrados e criados em pastagem que apresentam menor deposição de gordura. Apesar do escore de marmorização superior ao encontrado por Cedrés et al. (2002), aos 150 dias de confinamento, os bubalinos castrados apresentaram pouca deposição de gordura intramuscular.

Não foram encontradas diferenças significativas $(\mathrm{P}>0,05)$ entre os períodos de confinamento para os parâmetros sensoriais avaliados no painel (Tabela 4). Tatum et al. (1980) também não observaram diferenças no sabor, na suculência e na maciez da carne de bovinos abatidos aos 100, 130 e 160 dias de confinamento.

A carne bubalina nos períodos de confinamento avaliados não apresentou sabor estranho, aroma estranho nem cor escura. Para o consumidor, a cor da carne fresca é vermelho brilhante (Cross, 1994). Pelo painel sensorial, a carne bubalina apresentou coloração próxima ao vermelho brilhante.

Segundo Oliveira (2005), há um conceito de que a carne bubalina é escura porque os animais são abatidos em idade avançada. Neste estudo, a carne bubalina não apresentou cor escura, provavelmente porque era de animais jovens com idade entre 18 meses (animais abatidos aos 75 dias de confinamento) e 21 meses (animais abatidos aos 150 dias de confinamento), pois, de acordo com Felício (2000), a carne de animais terminados em confinamento e abatidos aos 18 e 24 meses é mais clara, semelhante à encontrada em supermercados de países de clima temperado.

Os valores obtidos para cor da carne bubalina foram semelhantes aos encontrados por Manço (2006) na carne de
Tabela 4 - Características sensoriais da carne, avaliadas pelo painel, de bubalinos da raça Murrah castrados abatidos aos 75 e 150 dias de confinamento

\begin{tabular}{|c|c|c|}
\hline \multirow[t]{2}{*}{ Parâmetro } & \multicolumn{2}{|c|}{ Período em confinamento } \\
\hline & 75 & 150 \\
\hline Aroma & $6,33 \pm 0,89$ & $6,55 \pm 0,86$ \\
\hline Aroma estranho & $1,15 \pm 0,37$ & $1,05 \pm 0,24$ \\
\hline Sabor & $6,71 \pm 1,20$ & $6,81 \pm 1,20$ \\
\hline Sabor estranho & $1,10 \pm 0,31$ & $1,00 \pm 0,00$ \\
\hline Maciez & $4,19 \pm 1,42$ & $4,15 \pm 0,88$ \\
\hline Suculência & $5,95 \pm 0,62$ & $5,56 \pm 0,94$ \\
\hline Mastigabilidade & $5,56 \pm 1,98$ & $5,97 \pm 1,82$ \\
\hline Cor & $3,50 \pm 1,67$ & $4,16 \pm 1,82$ \\
\hline
\end{tabular}

* Médias seguidas de letras distintas diferem $(P<0,05)$ entre si.

bovinos Nelore abatidos aos 2 anos de idade maturada por dois dias $(3,85)$

Monin \& Ouali (1991) relataram que o aumento na idade ou no peso de abate intensifica a cor da carne, em virtude do aumento do nível de mioglobina. No entanto, neste estudo não foi constatada diferença na cor quando o período de confinamento foi maior.

O aroma, quando avaliado na escala não estruturada de nove pontos, foi próximo ao aroma característico da carne bovina e superior ao encontrado por Manço (2006), em bovinos da raça Nelore com 2 anos de idade.

O valor obtido para maciez avaliada pelo painel sensorial confirmou a maciez avaliada pela força de cisalhamento e a avaliada pelo índice de força de cisalhamento. No painel sensorial, a carne foi julgada pelos provadores que atribuíram notas 4,19 para os 75 dias de confinamento e 4,15 para 150 dias de confinamento. Na escala fornecida aos provadores, a nota 4 é a estipulada para a carne macia.

No painel sensorial, a suculência ficou entre a escala nem seco nem suculento e levemente suculento, prejudicada pelo baixo escore de marmorização (Tabela 3), pois, quanto mais alto o escore de marmorização, maior a sensação de suculência da carne (Monin \& Ouali, 1991; Cross, 1994; Roça, 1997). Os valores de suculência foram superiores aos achados por Manço (2006), que constatou nota 4,60.

A mastigabilidade é um atributo secundário da textura, avaliado pelo número de mastigadas necessárias para deixar a carne em condições de ser deglutida (Roça, 1997). No painel sensorial, esta característica foi avaliada pela escala não estruturada de 1 (elástica borrachenta e difícil de deglutir) a 9 pontos (desintegra facilmente na boca, fácil de deglutir). A carne de búfalo apresentou boa mastigabilidade avaliada pelos provadores, com classificação de 5,56 a 5,97, superior à encontrada por Manço (2006), que observou 3,98 para bovinos da raça Nelore. 


\section{Conclusões}

O tempo de confinamento não influencia as características físico-químicas e sensoriais da carne de bubalinos jovens da raça Murrah, com exceção do escore de marmorização, que é menor aos 75 dias de confinamento. A carne bubalina foi classificada como macia em todos os períodos de confinamento, tanto nas análises de força de cisalhamento, como pelo índice de fragmentação miofibrilar e pelo painel sensorial. Bubalinos abatidos aos 75 e 150 dias de confinamento não apresentam carne com aroma e sabor estranho nem cor escura no painel sensorial, portanto, o abate de bubalinos jovens deve ser fomentado para produção de carne de excelente qualidade sensorial.

\section{Literatura Citada}

ALLINGHAM, P.G.; HARPER, G.S.; HUNTER, R.A. Effect of growth path on the tenderness of semitendinosus muscle of Brahman-cross steers. Meat Science, v.48, p.65-73, 1998.

ANDRighetTO, C.; JORGE, A.M.; CALiXTO, M.G. et al. Características qualitativas da carcaça e da carne de bubalinos mediterrâneo terminados em confinamento e abatidos em diferentes pesos. In: CONGRESSO BRASILEIRO DE CIÊNCIA E TECNOLOGIA DA CARNE, 3., 2005, São Pedro. Anais... São Paulo: Instituto de Tecnologia de Alimentos, 2005. (CD-ROM).

ASSOCIATION OF OFFICIAL ANALYTICAL CHEMISTS - AOAC. Official methods of analysis. 15.ed. Arlington: 1990. 1298p.

BERG, R.T.; WALTERS, R.M. The meat animal: changes and changenles. Journal of Animal Science, v.57, n.2, p.133-146, 1983.

BERNARDES, O. Os Búfalos no Brasil. In: SIMPÓSIO DE BÚFALO DE LAS AMÉRICAS, 2., SIMPÓSIO EUROPA-AMERICA, 2., 2006, Medellín. Anais... Medellín: 2006. v.3, p.18-23.

CEDRÉS, J.F.; CRUDELI, G.A.; PATIÑO, E.M. et. al. [2002]. Composición química y características físicas de la carne de búfalos criados en forma extensiva en la provincia de Formosa. Disponível em: <http://www.unne.edu.ar/Web/cyt/cyt/2002/cyt.htm> Acesso em: 4/8/2008.

CORRÊA, A.; TRAMOSO, E. Búfalos. Revista Produz. v.1, n.6, p.36-43, 2004.

CROSS, H.R. Características organolépticas de la carne. In: PRICE, J.F.; SCHWEIRGERT, B.S. (Eds.). Ciência de la carne y de los productos carneaos. Acribia, 1994. p.279-294.

CULLER, R.D.; PARRISH JR., F.C.; SMITH, G.G. et al. Relationship of myofibril fragmentation index to certain chemical, physical and sensory characteristics of bovine Longissimus muscle. Journal of Food Science, v.43, p.1177-1180, 1978.

DABÉS, A.C. Maturação da carne bovina: alterações estruturais. Revista Nacional da Carne, v.24, n.283, p.66-71, 2000.

FELÍCIO, P.E. Qualidade da carne Nelore e o mercado mundial. In: SEMINÁRIO DO PMGRN, 9., 2000, Ribeirão Preto. Palestras... Ribeirão Preto: Faculdade de Medicina de Ribeirão Preto, Universidade de São Paulo, 2000. p.1-10.

FELÍCIO, P.E. Fatores ante e post mortem que influenciam na qualidade da carne bovina. In: PEIXOTO, A.M.; MOURA, J.C.; FARIA, V.P. (Eds.) Produção do novilho de corte. Piracicaba: Fundação de Estudos Agrários "Luis de Queiroz", 1997. p.79-97.

JORGE, A.M. Desempenho em confinamento e características de carcaça em bubalinos. In: SIMPÓSIO PAULISTA DE
BUBALINOCULTURA, 1999, Jaboticabal. Anais... Jaboticabal: Funep, 1999. v.1. p.51-67.

MANÇO, M.C.W. Características físico-químicas, sensoriais e higiênicas da carne bovina em duas classes de maturidade e sob influência da maturação. Botucatu: Universidade Estadual Paulista, 2006. 124p. Tese (Doutorado em Zootecnia) Universidade Estadual Paulista, 2006.

MATTOS, J.C.A.; NOGUEIRA, J.R.; OLIVEIRA, A.A.D. et al. Comparasion on carcass, meat cuts and some meat quality characteristis of buffaloes and of zebu In: CONGRESSO MUNDIAL DE CRIADORES DE BÚFALOS, 6., 1997, Caserta. Proceedings... Caserta: 1997. p.442-446.

MEILGAARD, M.; CIVILLE, G.V.; CARR, B.T. Sensory evaluation techniques. Boca Raton: CRC Press, 1990. 281p.

MONIN, G.; OUALI, A. Muscle differentiation and meat quality. In: RALSTON, L. (Ed.) Developments in meat science. Elsevier Applied Science, 1991. p.89-138.

NATIONAL RESEARCH COUNCIL - NRC. Nutrient requeriments of beef cattle. 7.ed. Washington D.C., 1996. 242p.

NÚCLEO DE ESTUDOS E PESQUISAS EM ALIMENTAÇÃO NEPA - UNICAMP. Tabela brasieleira de composição de alimentos - versão 1. Campinas: 2004. 44p.

OLIVEIRA, A.L. Búfalos: produção, qualidade de carcaça e de carne. Alguns aspectos quantitativos qualitativos e nutricionais para produção do melhoramento genético. Revista Brasileira de Reprodução Animal, v.29, n.2, p.122-134, 2005

PURCHAS, R.W.; BURNHAM, D.L.; MORRIS, S.T. Effects of growth potential and growth path on tenderness of beef longissimus muscle from bulls and steers. Journal of Animal Science, v.80, p.3211-3221, 2002.

ROÇA, R.O. Tecnologia da carne e produtos derivados. Botucatu: Departamento de Gestão e Tecnologia Agroindustrial, FCA, UNESP, 1997. 205p.

ROÇA, R.O.; SERRANO, A.M.; BONASSI, I.A. Utilização de toucinho na elaboração de fiambres com carne de frango. Ciência e Tecnologia de Alimentos, v.8, n.1, p.67-76, 1988.

ROÇA, R.O.; BONASSI, I.A. Seleção de provadores para produtos cárneos. In: CONGRESSO BRASILEIRO DE CIÊNCIA E TECNOLOGIA DE ALIMENTOS, 7., 1985, Itabuna/Ilhéus. Anais... Itabuna/Ilhéus: SBCTA, 1985. p.83.

SPANGHERO, M.; GRACCO, L.; VALUSSO, R. et al. In vivo performance, slaughtering traits and meat quality of bovine (Italian Simmental) and buffalo (Italian Mediterranean) bulls Livestock Production Science, v.7, p.1-13, 2004.

UNITED STATES DEPARTMENT OF AGRICULTURE - USDA. Official United States standards for grades of carcass beef. Washington, D.C.: MAS-USDA, 1997.

UNIVERSIDADE FEDERAL DE VIÇOSA - UFV. SAEG Sistema de Análise Estatística e Genética. Viçosa, MG: 1997. (CD-ROM).

TATUM, J.D.; SMITH, G.C.; BERRY, B.W. et al. Carcass characteristics, time on feed and cooked beef palatability attributes. Journal of Animal Science, v.50, n.5, p.833-840, 1980.

VAZ, F.N.; RESTLE, J.; BRONDANI, I.L. et al. Estudo da carcaça e da carne de bubalinos Mediterrâneo terminados em confinamento com diferentes fontes de volumoso. Revista Brasileira de Zootecnia, v.32. n.2, p.393-404, 2003.

VESTERGAARD, M.; THERKILDSEN, M.; HENCKEL, P. et al. Influence of feeding intensity, grading and finishing feeding on meat and eating quality of young bulls and relationship between muscle fibre characteristicas, fibre fragmentation and meat tenderness. Meat Science, v.54, p.187-195, 2000.

WHEELER, T.L.; KOOHMARAIE, M.; SHACKELFORD, S.D. Standardized Warner-Bratzler shear force procedures for meat tenderness measurement. Clay Center: Roman L. Hruska U. S. MARC. USDA, 1995. 7p. 